\title{
Meta-analysis of two randomized phase III trials (TCOG GI-0801 and ECRIN TRICS) of biweekly irinotecan plus cisplatin versus irinotecan alone as second-line treatment for advanced gastric cancer
}

\author{
Kazuhiro Nishikawa ${ }^{1}$ - Wasaburo Koizumi ${ }^{2} \cdot$ Akira Tsuburaya $^{3} \cdot$ Takeharu Yamanaka $^{4} \cdot$ Satoshi Morita $^{5}$. \\ Kazumasa Fujitani ${ }^{6} \cdot$ Yusuke Akamaru $^{7} \cdot$ Ken Shimada $^{8} \cdot$ Hisashi Hosaka $^{9} \cdot$ Norisuke Nakayama $^{10}$. \\ Toshimasa Tsujinaka ${ }^{11}$. Junichi Sakamoto ${ }^{12}$
}

Received: 16 April 2019 / Accepted: 6 July 2019 / Published online: 15 July 2019

(c) The International Gastric Cancer Association and The Japanese Gastric Cancer Association 2019

\begin{abstract}
Background Biweekly irinotecan (CPT-11) plus cisplatin (CDDP) combination (BIRIP) and CPT-11 alone are both expectable options for treating advanced gastric cancer (AGC) in a second-line setting. We conducted a meta-analysis to compare the efficacy and safety of these two regimens in patients enrolled two randomized phase III trials.

Patients and methods Individual patient-level data from two randomized phase III trials were collected for this study. In both trials, patients with AGC refractory to S-1-based chemotherapy were randomly allocated to BIRIP (CPT-11, $60 \mathrm{mg}$ / $\mathrm{m}^{2}$; CDDP, $\left.30 \mathrm{mg} / \mathrm{m}^{2}, \mathrm{q} 2 \mathrm{w}\right)$ or to CPT-11 $\left(150 \mathrm{mg} / \mathrm{m}^{2}, \mathrm{q} 2 \mathrm{w}\right)$.

Results Cumulative data from 290 eligible patients were evaluated. The OS was 12.3 months [95\% confidence interval (CI) 10.5-14.1] in the BIRIP group and 11.3 months (95\% CI 10.0-13.2) in the CPT-11 group (hazard ratio $0.87 ; 95 \%$ CI $0.68-1.12, P=0.272$ ), while PFS was significantly longer in the BIRIP group (4.3 months [95\% CI 3.5-5.1]) than in the CPT-11 group (3.3 months [2.9-4.1]; HR 0.77; 95\% CI 0.61-0.98, $P=0.035)$. The response rate was $20.5 \%$ in the BIRIP group and $16.0 \%$ in the CPT-11 group $(P=0.361)$. However, the disease control rate was significantly better in the BIRIP group $(72.1 \%)$ than in the CPT-11 group $(59.2 \%)(P=0.032)$. The two groups did not differ significantly in the incidences of grade 3 or worse adverse events.

Conclusions Both BIRIP and CPT-11 may be good therapeutic options for patients with AGC as second-line treatment.

Clinical trial registration UMIN 000025367.
\end{abstract}

Keywords Meta-analysis · Advanced gastric cancer · Second-line chemotherapy · Biweekly irinotecan plus cisplatin · Irinotecan

\section{Introduction}

Gastric cancer is one of the leading causes of cancerrelated deaths worldwide [1]. In general, combination chemotherapy regimens provide better response rates (RRs) and modestly improved survival benefits compared

Kazuhiro Nishikawa and Wasaburo Koizumi contributed equally to this work.

Kazuhiro Nishikawa

kazuno13@hotmail.co.jp;

nishikawa.kazuhiro.mp@mail.hosp.go.jp

Extended author information available on the last page of the article with single-agent therapies for advanced gastric cancer (AGC) [2]. However, after undergoing first-line chemotherapy, most patients will eventually require second-line chemotherapy (SLC), mainly as a result of tumor progression. In addition, many patients who relapse during or soon after adjuvant chemotherapy must undergo SLC after recurrence $[3,4]$. Three trials demonstrated prolongation of survival with SLC (irinotecan or docetaxel monotherapy) over best supportive care [5-7]. A meta-analysis of these trials supported the efficacy of SLC in the treatment of AGC [8], and showed that salvage chemotherapy was associated with a significant reduction in the risk of death with [hazard ratio (HR) 0.55 and 0.71 for irinotecan and 
docetaxel, respectively]. Irinotecan (CPT-11) monotherapy was used as the active comparator in several randomized trials for AGC as the SLC [9-12]. And randomized phase III trials compared treatment with weekly paclitaxel and biweekly CPT-11 in patients with AGC as the SLC were reported that both are reasonable SLC options [13, 14]. Based on these results, CPT-11 are considered to be a good option for AGC as the SLC.

Biweekly irinotecan plus cisplatin (CDDP) combination therapy (BIRIP) was developed to reduce CPT-11-associated diarrhea and febrile neutropenia by decreasing the dose of CPT-11 [15, 16], and showed promising efficacy and a manageable toxicity profile [16]. Two randomized trials both compared BIRIP versus CPT-11 alone in the second-line setting $[17,18]$. In the TCOG GI-0801 trial, Higuchi et al. reported $(n=130)$ that BIRIP significantly prolonged progression-free survival (PFS) (HR 0.68) compared with CPT-11 monotherapy, although it did not have an overall survival (OS) benefit (HR: 1.00) in patients with metastatic or recurrent gastric cancer that progressed after S-1-based chemotherapy [17]. Nishikawa et al. conducted the TRICS trial and reported $(n=168)$ that BIRIP did not show a significant benefit in terms of OS (HR 0.83) or PFS (HR 0.86) compared with CPT-11 alone in patients with progressive AGC who were previously treated with S-1 monotherapy [18]. Although BIRIP might be a promising regimen as SLC to replace CPT-11 monotherapy, the findings of the two aforementioned trials are limited as BIRIP showed no OS benefit and there were no differences in response rates between the two regimens, most likely because the analyses were underpowered. Therefore, it still remains unclear whether BIRIP is effective in patients with AGC in a second-line setting.

Therefore, we conducted a meta-analysis to evaluate the safety and efficacy of BIRIP for AGC as the SLC.

\section{Patients and methods}

\section{Study design}

This meta-analysis follows PRISMA-IPD [19]. We included all completed and peer review published randomized clinical trials that investigated the effect of irinotecan plus cisplatin combination therapy (BIRIP) treated as second-line chemotherapy for the treatment of gastric and gastroesphageal adenocarcinoma. We searched PubMed and the Cochrane Central Register of Controlled Trials up to the date of 31 March 2019. The keywords were "Gastric Cancer OR Gastric Carcinoma OR Gastroesophageal Junction Adenocarcinoma" AND "CPT-11 OR Irinotecan" AND "CDDP OR Cisplatin" AND "Refractory OR Second-line". The following were the inclusion criteria: (1) Phase II/III randomized controlled clinical trials (RCTs); (2) Pathological diagnosis of AGC or gastroesophageal junction adenocarcinoma; (3) First-line fluorouracil-containing chemotherapy failed; (4) Second-line treatment regimen was irinotecan plus cisplatin combination therapy.

We selected two randomized phase III trials, the TCOG GI-0801 trial and the TRICS trial (Fig. 1). These two randomized trials both compared BIRIP versus CPT-11 alone, and employed the same regimen. Having obtained individual patient-level data (IPD), we have made it possible to evaluate in detail. Furthermore, the eligibility criteria were almost equivalent and the treatment methods were identical. In both trials, patients were randomly allocated to BIRIP (CPT-11, $60 \mathrm{mg} / \mathrm{m}^{2}$; CDDP, $30 \mathrm{mg} / \mathrm{m}^{2}, \mathrm{q} 2 \mathrm{w}$ ) or to CPT11 monotherapy $\left(150 \mathrm{mg} / \mathrm{m}^{2}, \mathrm{q} 2 \mathrm{w}\right)$. The dose of CPT-11 was chosen according to physician preference and was not based on the presence or absence of the UGT1A1 genotype. In terms of patient enrollment, inclusion and exclusion criteria were reported previously $[17,18]$. Briefly, patients
Fig. 1 PRISMA flow diagram of the literature search

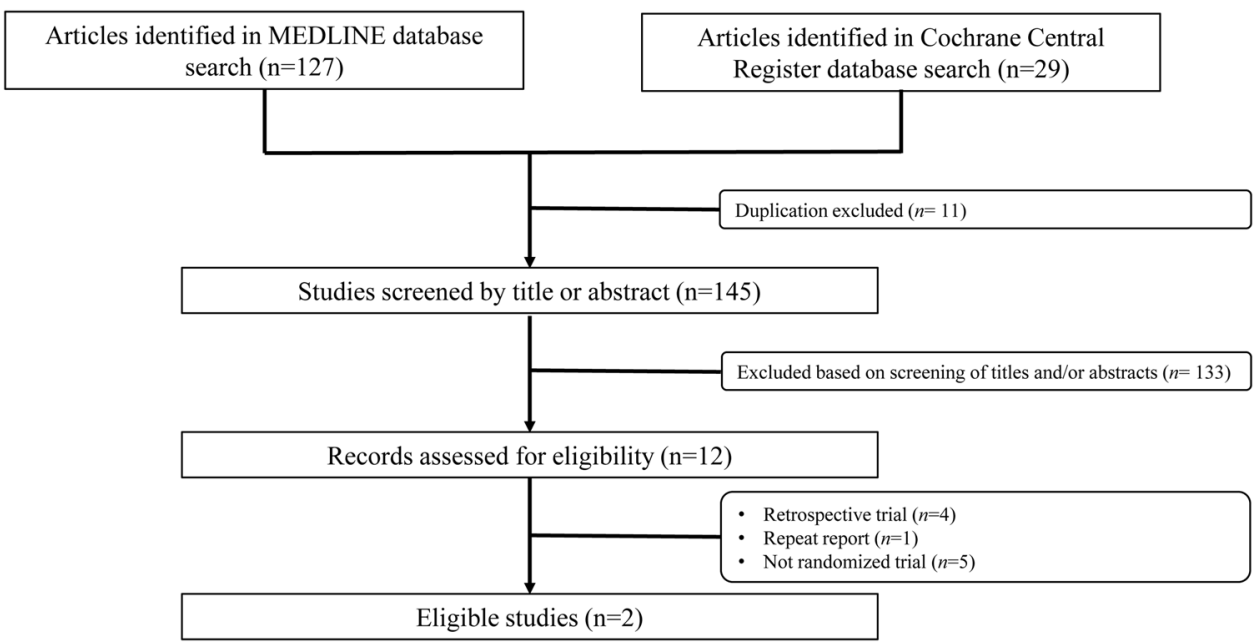


with histologically confirmed AGC were eligible that were refractory to the first-line S-1-based chemotherapy (TCOG GI-0801) or S-1 monotherapy (TRICS), or that recurred during or within 6 months after the completion of adjuvant therapy with S-1. Other eligibility criteria were follow: patients aged $\geq 20$ years; an estimated life expectancy of at least 12 weeks; written informed consent; an Eastern Cooperative Oncology Group performance status (PS) of 2 or less (TCOG GI-0801), 1 or less (TRICS); with adequate organ function (white blood cell count $\geq 4000 / \mathrm{mm}^{3}$ and $\leq 12,000 \mathrm{~mm}^{3}$, neutrophil count $\geq 2000 / \mathrm{mm}^{3}$, platelet count $\geq 100,000 / \mathrm{mm}^{3}$, hemoglobin level $\geq 8.0 \mathrm{~g} / \mathrm{dL}$, aspartate aminotransferase and alanine aminotransferase (ALT) levels $\leq 100 \mathrm{IU} / \mathrm{L}$, total bilirubin level $\leq 1.50 \mathrm{mg} / \mathrm{dL}$, and creatinine level $\leq$ upper limit of normal (TCOG GI-0801), $\leq 1.20 \mathrm{mg} / \mathrm{dL}$ (TRICS)). Exclusion criteria included, history of anti-tumor therapy (except for S-1-based chemotherapy and surgery), additional malignancies, or significant comorbidities.

We first verified the integrity of IPD from the TCOG GI-0801 and TRICS trials. All clinical data were extracted and held centrally at the data center of the Epidemiological $\&$ Clinical Research Information Network (ECRIN), a nonprofit organization.

This meta-analysis was conducted in accordance with the Declaration of Helsinki and registered with UMIN-CTR, UMIN 000025367. All patients in the TCOG GI-0801 and TRICS trials provided written informed consent after being informed about the purpose and investigational nature of the respective studies. The institutional review boards or ethics committees of all participating centers reviewed and approved the protocol.

\section{Assessment}

The primary endpoint of this study was OS. The secondary endpoints were PFS, overall response rate (ORR), disease control rate (DCR), and adverse events (AEs). Tumor responses were analyzed in patients with at least one measurable lesion at baseline using Response Evaluation Criteria in Solid Tumors (RECIST) version 1.0. Tumor responses were classified as complete response (CR), partial response $(\mathrm{PR})$, stable disease (SD), or progressive disease (PD). The ORR was defined as the proportion of patients with CR or $\mathrm{PR}$, and the DCR was defined as the proportion of patients with CR, PR, or SD. Grade 3 or worse adverse events were evaluated using the Common Terminology Criteria for Adverse Events (CTCAE) version 3.0.

\section{Statistical methods}

The OS and PFS curves were constructed as time-to-event plots using the Kaplan-Meier method. Time-to-event curves were compared using log-rank tests and hazard ratios (HRs) were estimated by Cox regression models. The confidence coefficient for the confidence intervals of the median OS, PFS, and HR was set to $95 \%(P<0.05)$. The chi-square test and two-sided $95 \%$ CI were used to compare the ORR and DCR between the two groups. All clinical data were held centrally at the ECRIN data center and analyzed using SAS for Windows version 9.3 (SAS Institute, Cary, NC, USA).

\section{Results}

\section{Patient characteristics}

We extracted data of 127 patients eligible for the TCOG GI-0801 trial $(N=130)$, and 163 patients eligible for TRICS trial $(N=168)$. Thus cumulative data of 290 eligible patients were evaluated. One hundred and forty-five patients (50\%) were allocated to the BIRIP group and $145(50 \%)$ were allocated to the CPT-11 group. Both arms were well balanced in terms of baseline clinical characteristics (Table 1).

\section{Survival}

The OS was 12.3 months (95\% confidence interval [CI]: 10.5-14.1) in the BIRIP group and 11.3 months (95\% CI 10.0-13.2) in the CPT-11 group [hazard ratio (HR) 0.87; 95\% CI 0.68-1.12, $P=0.272$ ] (Fig. 2a). Furthermore, PFS was significantly longer in the BIRIP group (4.3 months [95\% CI 3.5-5.1]) than in the CPT-11 group (3.3 months [95\% CI 2.9-4.1]; HR 0.77; 95\% CI 0.61-0.98, $P=0.035$ ) (Fig. 2b).

Exploratory subgroup analyses in the BIRIP group showed positive associations between OS and male gender, intestinal histological type, and primary-site disease (Fig. 3). Tests for interaction in the BIRIP group showed a treatment effect only for male gender $(P=0.011)$.

\section{Tumor responses}

Of the 122 patients in the BIRIP group who had measurable disease, three achieved CR, 22 achieved PR, and 63 achieved SD. Of the 125 patients in the CPT-11 group who had measurable disease, one achieved CR, 19 achieved PR, and 54 achieved SD. The ORR was 20.5\% [95\% CI 13.3-27.7] in the BIRIP group and $16.0 \%$ [95\% CI 9.6-22.4] in the CPT11 group, indicating no significant difference $(P=0.361)$ (Table 2). However, the DCR was significantly higher in the BIRIP group (72.1\% [95\% CI 64.2-80.1]) than in the CPT11 group (59.2\% [95\% CI 50.6-67.8]) $(P=0.032)$ (Table 2). 
Table 1 Patient characteristics

\begin{tabular}{|c|c|c|c|}
\hline Eligible patients, $n=290$ & BIRIP & CPT-11 & $P$ value \\
\hline No. of patients (\%) & $145(50)$ & $145(50)$ & \\
\hline \multicolumn{4}{|l|}{ Age } \\
\hline Median (range) & $66(29-85)$ & $67(35-87)$ & 0.189 \\
\hline$<65$ & $62(42.8)$ & $54(37.2)$ & 0.338 \\
\hline$\geq 65$ & $83(57.2)$ & $91(62.8)$ & \\
\hline \multicolumn{4}{|l|}{ Gender } \\
\hline Male & $114(78.6)$ & $117(80.7)$ & 0.662 \\
\hline Female & $31(21.4)$ & $28(19.3)$ & \\
\hline \multicolumn{4}{|l|}{ ECOG PS } \\
\hline 0 & $111(76.6)$ & $109(75.2)$ & 0.784 \\
\hline 1 & $34(23.4)$ & $36(24.8)$ & \\
\hline \multicolumn{4}{|l|}{ Histology } \\
\hline Intestinal & $76(52.4)$ & $69(47.6)$ & 0.411 \\
\hline Diffuse & $69(47.6)$ & $76(52.4)$ & \\
\hline \multicolumn{4}{|l|}{ Disease status } \\
\hline Progression & $65(44.8)$ & $62(42.8)$ & 0.723 \\
\hline Recurrence & $80(55.2)$ & $83(57.2)$ & \\
\hline \multicolumn{4}{|l|}{ Primary site } \\
\hline No & $89(61.4)$ & $98(67.6)$ & 0.269 \\
\hline Yes & $56(38.6)$ & $47(32.4)$ & \\
\hline \multicolumn{4}{|l|}{ Prior platinum agent } \\
\hline No & $109(75.2)$ & $109(75.2)$ & 1.000 \\
\hline Yes & $\begin{array}{l}36(24.8) \\
\text { Cisplatin } 33 \\
\text { Oxaliplatin } 3\end{array}$ & $\begin{array}{l}36(24.8) \\
\text { Cisplatin } 35 \\
\text { Oxaliplatin } 1\end{array}$ & \\
\hline \multicolumn{4}{|l|}{ Measurable lesion } \\
\hline No & $23(15.9)$ & $20(13.8)$ & 0.620 \\
\hline Yes & $122(84.1)$ & $125(86.2)$ & \\
\hline \multicolumn{4}{|l|}{ Peritoneal metastasis } \\
\hline No & $128(88.3)$ & $123(84.8)$ & 0.389 \\
\hline Yes & $17(11.7)$ & $22(15.2)$ & \\
\hline
\end{tabular}

BIRIP biweekly irinotecan plus cisplatin, CPT-11 irinotecan, ECOG Eastern Cooperative Oncology Group, $P S$ performance status

\section{Adverse events}

The proportions of patients with adverse events are shown in Table 3. The proportions of patients with grade 3 or worse adverse events did not differ between the two groups. For example, neutropenia and elevated serum creatinine levels occurred in $35.9 \%$ and $0.7 \%$ of patients in the BIRIP group, respectively, compared with $32.4 \%$ and $0.7 \%$ of patients in the CPT-11 group, respectively. Grade 3 or worse diarrhea ( $1.4 \%$ versus $4.1 \%$, respectively) and febrile neutropenia $(0 \%$ versus $2.1 \%$, respectively) were rarely observed in the BIRIP group. Although the incidence of all grade leukopenia, neutropenia, thrombocytopenia and creatinine increased were higher in BIRIP group than the CPT-11 group, all grade diarrhea was more common in CPT-11 group.

\section{Discussion}

This meta-analysis is the first report comparing the efficacy and safety of biweekly BIRIP to those of CPT-11 monotherapy in a large sample. While BIRIP was not superior to CPT-11 monotherapy in terms of OS and ORR, the BIRIP group demonstrated significantly longer PFS than the CPT11 group (HR 0.77; 95\% CI 0.61-0.98, $P=0.035$ ), as well as a significantly higher disease control rate $(P=0.032)$. Moreover, patients receiving BIRIP rarely developed grade 3 or worse diarrhea (1.4\%) and none developed febrile neutropenia $(0 \%)$.

While our meta-analysis failed to demonstrate differences in either OS or ORR between the two groups, it was meaningful to be able to prove the superiority of BIRIP over CPT-11 monotherapy in terms of PFS. In Japan, more than $70 \%$ of patients with AGC receive third-line chemotherapy. It is considered difficult to demonstrate the superiority of a second-line regimen in terms of OS, because OS is strongly affected by third or further line treatments. Indeed, in this meta-analysis, about $72 \%$ of patients in both groups received third-line chemotherapy.

Our subgroup analysis of OS showed no interaction between the patients with or without prior platinum administration. In this meta-analysis, post-protocol therapy was administered in $73 \%(106 / 145)$ and $72 \%(104 / 145)$ of patients in the BIRIP and CPT-11 monotherapy, respectively. We could not obtain detailed data up to the final-line because of still under post-treatment, at least $12.9 \%(27 / 210)$ of the post-treatment patients received chemotherapy with platinum agents. This "crossover" might be effected for no interaction.

It is unknown why BIRIP therapy resulted in superior $\mathrm{OS}$ in males than in females. There are no reports of gender differences affecting the efficacy of irinotecan in advanced cancer. It is also unclear why longer OS was associated with intestinal histological type and primary-site disease in patients treated with BIRIP, though the effect of additional platinum may have been beneficial.

Irinotecan is activated by hydrolysis to $\mathrm{SN}-38$, a potent topoisomerase I inhibitor that is primarily inactivated through biotransformation into SN-38 glucuronide (SN$38 \mathrm{G})$ by UGT1A1 [20, 21]. It was reported that interpatient differences in SN-38G formation were correlated with the occurrence of severe diarrhea [22], and that UGT1A1 genotype was strongly associated with severe neutropenia in cancer patients receiving irinotecan [23]. In our metaanalysis, the dose of irinotecan was lower in the BIRIP group $\left(60 \mathrm{mg} / \mathrm{m}^{2} / \mathrm{q} 2 \mathrm{w}\right)$ than in the CPT-11 group $(150 \mathrm{mg} /$ $\left.\mathrm{m}^{2} / \mathrm{q} 2 \mathrm{w}\right)$. Importantly, BIRIP was well tolerated, with no cases of febrile neutropenia and a low rate of grade 3 or worse irinotecan-associated diarrhea. These results suggest 
a

OS

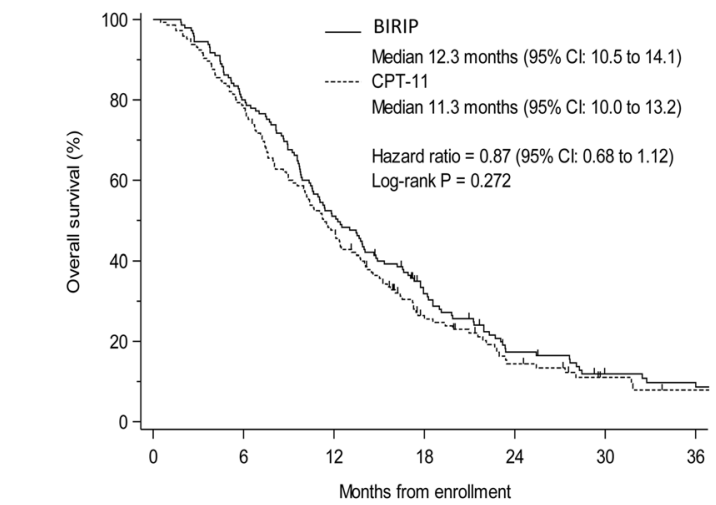

No. at risk b

PFS

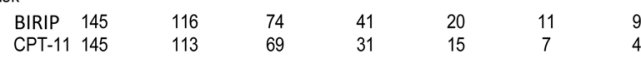

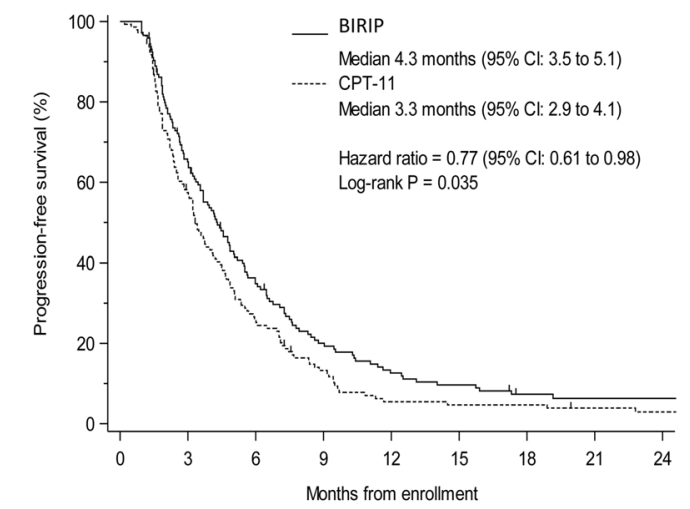

No. at risk

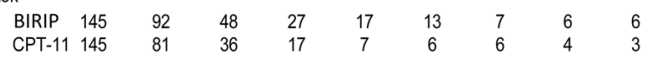

Fig. 2 Kaplan-Meier curves for OS (a) and PFS (b) by treatment group. OS overall survival, PFS progression-free survival, BIRIP biweekly irinotecan plus cisplatin combination therapy, $C P T-11$ irinotecan

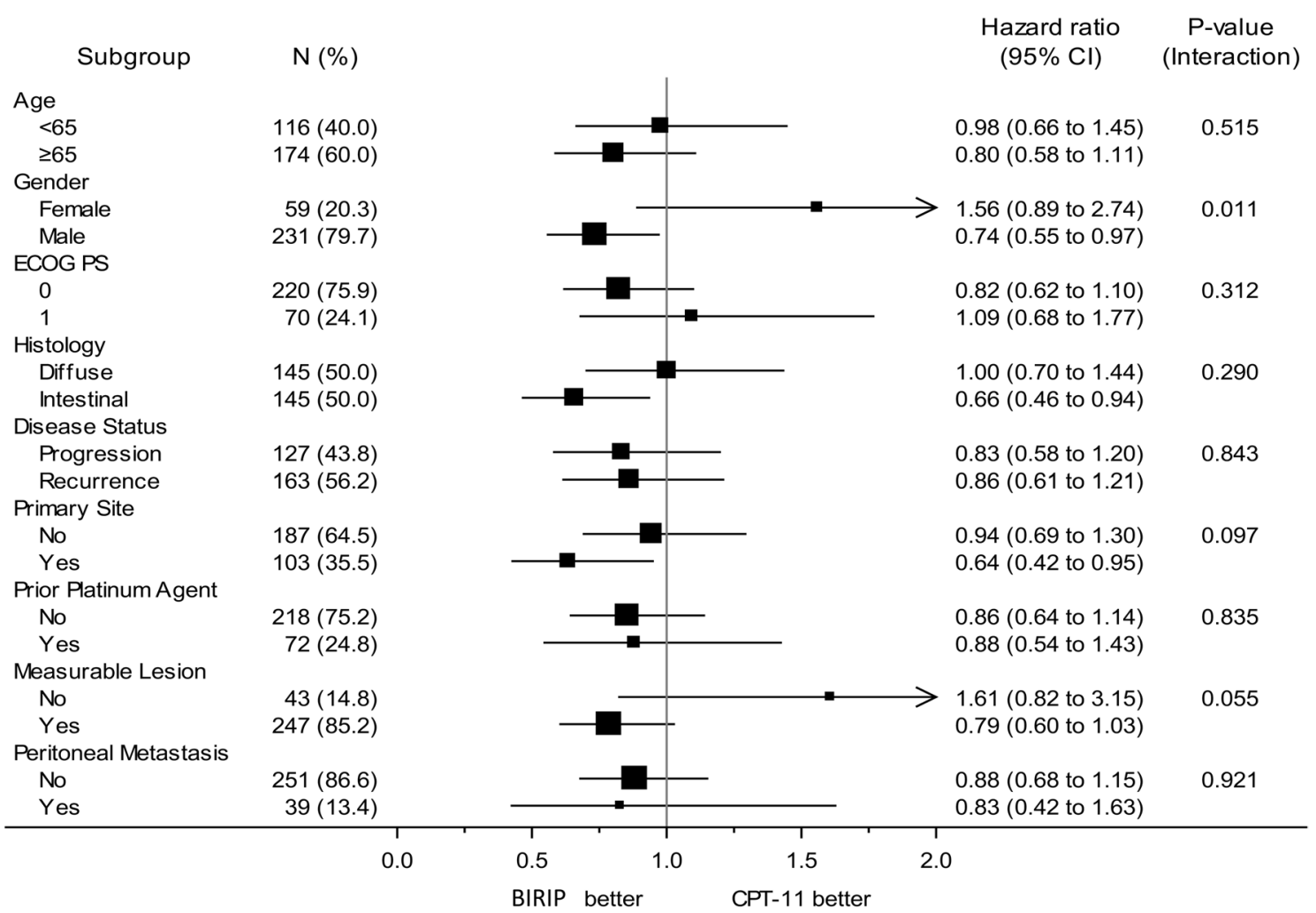

Fig. 3 Subgroup analysis for OS. ECOG Eastern Cooperative Oncology Group, PS performance status

that BIRIP might be an alternative to CPT-11 monotherapy as a SLC in patients with UGT1A1 polymorphism.

Shitara K et al. reported that each of the four classes of drugs used in the treatment of AGC (fluorouracil, platinum agents, taxanes, and irinotecan) was independently associated with improved OS [24]. These results suggest the importance of making these active agents available to all patients with AGC. A similar strategy is warranted for metastatic colorectal cancer, in which exposure to all three effective cytotoxic agents (fluorouracil, oxaliplatin, and irinotecan) was shown to prolong survival [25]. From this point of view, BIRIP may be a good treatment option 
Table 2 Clinical responses

\begin{tabular}{llll}
\hline & BIRIP & CPT-11 & $P$ value \\
\hline $\begin{array}{l}\text { No. of patients } \\
\text { Best overall response (\%) }\end{array}$ & 122 & 125 & \\
CR & $3(2.5)$ & $1(0.8)$ & \\
PR & $22(18.0)$ & $19(15.2)$ & \\
SD & $63(51.6)$ & $54(43.2)$ & \\
PD & $27(22.1)$ & $45(36.0)$ & \\
NE & $7(5.7)$ & $6(4.8)$ & \\
ORR (CR, PR) & $25(20.5)$ & $20(16.0)$ & 0.361 \\
$95 \%$ CI & $(13.3-27.7)$ & $(9.6-22.4)$ & \\
DCR (CR, PR, SD) & $88(72.1)$ & $74(59.2)$ & 0.032 \\
95\% CI & $(64.2-80.1)$ & $(50.6-67.8)$ & \\
\hline
\end{tabular}

$C R$ complete response, $P R$ partial response, $S D$ stable disease, $P D$ progressive disease, $N E$ not evaluable, $O R R$ overall response rate, $D C R$ disease control rate, $C I$ confidence interval

for patients treated without platinum agents at first-line chemotherapy.

Current standard adjuvant chemotherapy for Stage3 gastric cancer in Japan is regarded as S-1 plus docetaxel (DS) due to the JACCRO GC-07 trial [26]. As mentioned above, and in addition because some basic and clinical studies demonstrated a lack of cross resistance between docetaxel and paclitaxel, the platinum-based chemotherapy such as BIRIP may be good options for early relapse after adjuvant chemotherapy with DS.

There are several limitations to this study. This metaanalysis was not planned before the TCOG GI-0801 and TRICS trials were conducted, and was not designed to confirm the equivalent efficacy of the two regimens that were evaluated; in other words, the sample size was too small to conclude that BIRIP was clearly non-inferior to CPT-11 monotherapy, and thus the results should be interpreted with caution. Furthermore, application of the present results may be limited, as combination therapies containing platinum are currently the standard first-line treatment. Moreover, combination therapies containing platinum, such as CapeOX, are currently among the standard adjuvant treatments [27, 28]. Lastly, while the TCOG GI-0801 and TRICS trials were ongoing, the RAINBOW trial reported that ramucirumab plus paclitaxel combination therapy improved OS and PFS over weekly paclitaxel alone (OS, 9.6 vs. 7.4 months; PFS, 4.4 vs. 2.9 months, respectively) [29]. Thus, the combination of ramucirumab with paclitaxel is currently thought to be the standard second-line chemotherapy for AGC, and irinotecan monotherapy is considered as third or later line. As mentioned above, irinotecan and platinum are still active agents, so the findings in this manuscript can be applied partially in third- or later-line setting. Randomized trials as third- or later-line setting may be required to confirm our findings sure.

Table 3 Adverse events

\begin{tabular}{|c|c|c|c|c|c|c|}
\hline \multirow[t]{2}{*}{ Adverse events } & \multicolumn{2}{|c|}{$\operatorname{BIRIP}(n=145)$} & \multicolumn{2}{|c|}{ CPT-11 $(n=145)$} & \multicolumn{2}{|l|}{$P$ value } \\
\hline & $\begin{array}{l}\text { Any grade } \\
n(\%)\end{array}$ & $\begin{array}{l}\geq \mathrm{G} 3 \\
n(\%)\end{array}$ & $\begin{array}{l}\text { Any grade } \\
n(\%)\end{array}$ & $\begin{array}{l}\geq \mathrm{G} 3 \\
n(\%)\end{array}$ & Any grade & $\geq \mathrm{G} 3$ \\
\hline Leukopenia & $127(87.5)$ & $18(12.4)$ & $99(68.3)$ & $23(15.9)$ & $<0.0001$ & \\
\hline Neutropenia & $107(73.8)$ & $52(35.9)$ & $88(60.7)$ & $47(32.4)$ & 0.0174 & \\
\hline Anemia & $116(80.0)$ & $24(16.6)$ & $108(74.5)$ & $15(10.3)$ & & \\
\hline Thrombocytopenia & $52(35.9)$ & $1(0.7)$ & $25(17.2)$ & $1(0.7)$ & 0.0003 & \\
\hline Febrile neutropenia & - & $0(0.0)$ & - & $3(2.1)$ & - & \\
\hline AST increased & $49(33.8)$ & $5(3.4)$ & $58(40.0)$ & $7(4.8)$ & & \\
\hline ALT increased & $41(28.3)$ & $5(3.4)$ & $54(37.2)$ & $4(2.8)$ & & \\
\hline T-Bil increased & $15(10.3)$ & $3(2.1)$ & $26(17.9)$ & $3(2.1)$ & & \\
\hline ALP increased & $57(39.3)$ & $7(4.8)$ & $56(38.6)$ & $5(3.4)$ & & \\
\hline Creatinine increased & $39(26.9)$ & $1(0.7)$ & $15(10.3)$ & $1(0.7)$ & 0.0003 & \\
\hline Hyperkalemia & $50(34.4)$ & $1(0.7)$ & $52(35.9)$ & $3(2.1)$ & & \\
\hline Hyponatremia & $25(17.2)$ & $2(1.4)$ & $23(15.9)$ & $1(0.7)$ & & \\
\hline Fatigue & $46(31.7)$ & $9(6.2)$ & $37(25.5)$ & $6(4.1)$ & & \\
\hline Anorexia & $86(59.3)$ & $10(6.9)$ & $75(51.7)$ & $14(9.7)$ & & \\
\hline Nausea & $70(48.3)$ & $5(3.4)$ & $67(46.2)$ & $8(5.5)$ & & \\
\hline Vomiting & $38(26.2)$ & $1(0.7)$ & $35(24.1)$ & $3(2.1)$ & & \\
\hline Diarrhea & $33(22.8)$ & $2(1.4)$ & $65(44.8)$ & $6(4.1)$ & $<0.0001$ & \\
\hline Alopecia & $36(24.8)$ & - & 39 (26.9) & - & & \\
\hline
\end{tabular}

AST aspartate aminotransferase, $A L T$ alanine aminotransferase, $T$-Bil total bilirubin, $A L P$ alkaline phosphatase 
In conclusion, BIRIP significantly prolonged PFS compared with CPT-11 alone and was tolerated as second-line treatment for AGC, but did not prolong OS in this metaanalysis. Both BIRIP and CPT-11 may be good therapeutic options for this cohort of patients in Japan.

Acknowledgements We deeply appreciate all patients and investigators involved in the TCOG GI-0801 and TRICS trials. We also thank the Epidemiological \& Clinical Research Information Network (ECRIN) and the Tokyo Cooperative Oncology Group (TCOG) for their support.

Funding This work was supported, in part, by the non-profit organization Epidemiological \& Clinical Research Information Network (ECRIN) (no grant numbers apply).

\section{Compliance with ethical standards}

Conflict of interest Kazuhiro Nishikawa has received honoraria from Chugai, Taiho, Yakult, Eli Lilly, Tsumura, and EA Pharma, and research funding from Yakult and Taiho, outside the submitted work. Takeharu Yamanaka reports personal fees from Takeda, Taiho, Chugai and Boehringer, outside the submitted work. Satoshi Morita reports personal fees from Daiichi-Sankyo, outside the submitted work. Toshimasa Tsujinaka reports personal fees from Lilly, outside the submitted work. All remaining authors have declared no conflict of interest.

Ethical statements This trial was conducted in compliance with the ethical principles of the Declaration of Helsinki and the Ethical Guidelines for Clinical Studies of the Japanese Ministry of Health, Labour and Welfare. This trial was approved by the institutional review boards or ethics committees at all participating centers. This trial was registered with Clinical Trials.gov (UMIN 000025367).

\section{References}

1. Ferlay J, Soerjomataram I, Dikshit R, Eser S, Mathers C, Rebelo $\mathrm{M}$, et al. Cancer incidence and mortality worldwide: sources, methods and major patterns in GLOBOCAN 2012. Int J Cancer. 2015;136:E359-386.

2. Wagner AD, Grothe W, Haerting J, Kleber G, Grothey A, Fleig WE. Chemotherapy in advanced gastric cancer: a systematic review and meta-analysis based on aggregate data. J Clin Oncol. 2006;24:2903-9.

3. Sasako M, Sakuramoto S, Katai H, Kinoshita T, Furukawa $\mathrm{H}$, Yamaguchi $\mathrm{T}$, et al. Five-year outcomes of a randomized phase III trial comparing adjuvant chemotherapy with S-1 versus surgery alone in stage II or III gastric cancer. J Clin Oncol. 2011;29:4387-93.

4. Ito S, Ohashi Y, Sasako M. Survival after recurrence in patients with gastric cancer who receive S-1 adjuvant chemotherapy: exploratory analysis of the ACTS-GC trial. BMC Cancer. 2018; $18: 449$.

5. Thuss-Patience PC, Kretzschmar A, Bichev D, Deist T, Hinke A, Breithaupt K, et al. Survival advantage for irinotecan versus best supportive care as second-line chemotherapy in gastric cancer: A randomised phase III study of the Arbeitsgemeinschaft Internistische Onkologie (AIO). Eur J Cancer. 2011;47:2306-14.

6. Kang JH, Lee SI, Lim do H, Park KW, Oh SY, Kwon HC, et al. Salvage chemotherapy for pretreated gastric cancer: a randomized phase III trial comparing chemotherapy plus best supportive care with best supportive care alone. J Clin Oncol. 2012;30:1513-8.
7. Ford HE, Marshall A, Bridgewater JA, Janowitz T, Coxon FY, Wadsley J, et al. Docetaxel versus active symptom control for refractory oesophagogastric adenocarcinoma (COUGAR-02): an open-label, phase 3 randomised controlled trial. Lancet Oncol. 2014;15:78-86.

8. Kim HS, Kim HJ, Kim SY, Kim TY, Lee KW, Baek SK, et al. Second-line chemotherapy versus supportive cancer treatment in advanced gastric cancer: a meta-analysis. Ann Oncol. 2013;24:2850-4.

9. Roy AC, Park SR, Cunningham D, Kang YK, Chao Y, Chen LT, et al. A randomized phase II study of PEP02 (MM-398), irinotecan or docetaxel as a second-line therapy in patients with locally advanced or metastatic gastric or gastro-oesophageal junction adenocarcinoma. Ann Oncol. 2013;24:1567-73.

10. Sym SJ, Hong J, Park J, Cho EK, Lee JH, Park YH, et al. A randomized phase II study of biweekly irinotecan monotherapy or a combination of irinotecan plus 5-fluorouracil/leucovorin (mFOLFIRI) in patients with metastatic gastric adenocarcinoma refractory to or progressive after first-line chemotherapy. Cancer Chemother Pharmacol. 2013;71:481-8.

11. Tanabe K, Fujii M, Nishikawa K, Kunisaki C, Tsuji A, Matsuhashi N, et al. Phase II/III study of second-line chemotherapy comparing irinotecan-alone with $\mathrm{S}-1$ plus irinotecan in advanced gastric cancer refractory to first-line treatment with S-1 (JACCRO GC-05). Ann Oncol. 2015;26:1916-22.

12. Satoh T, Lee KH, Rha SY, Sasaki Y, Park SH, Komatsu Y, et al. Randomized phase II trial of nimotuzumab plus irinotecan versus irinotecan alone as second-line therapy for patients with advanced gastric cancer. Gastr Cancer. 2015;18:824-32.

13. Hironaka S, Ueda S, Yasui H, Nishina T, Tsuda M, Tsumura T, et al. Randomized, open-label, phase III study comparing irinotecan with paclitaxel in patients with advanced gastric cancer without severe peritoneal metastasis after failure of prior combination chemotherapy using fluoropyrimidine plus platinum: WJOG 4007 trial. J Clin Oncol. 2013;31:4438-44.

14. Lee KW, Maeng CH, Kim TY, Zang DY, Kim YH, Hwang IG, et al. A phase III study to compare the efficacy and safety of paclitaxel versus irinotecan in patients with metastatic or recurrent gastric cancer who failed in first-line therapy (KCSG ST1001). Oncologist. 2019;24:18-e24.

15. Sato A, Kurihara M, Matsukawa M, Shimada K, Yamazaki T, Nakamachi M, et al. Preliminary study of fortnightly irinotecan hydrochloride plus cisplatin therapy in patients with advanced gastric and colorectal cancer. Cancer Chemother Pharmacol. 2001;47:380-4.

16. Koizumi W, Kurihara M, Satoh A, Takiuchi H, Tanabe S, Shimada K, et al. Phase I/II study of bi-weekly irinotecan plus cisplatin in the treatment of advavced gastric cancer. Anticancer Res. 2005;25:1257-62.

17. Higuchi K, Tanabe S, Shimada K, Hosaka H, Sasaki E, Nakayama N, et al. Biweekly irinotecan plus cisplatin versus irinotecan alone as second-line treatment for advanced gastric cancer: a randomised phase III trial (TCOG GI-0801/BIRIP trial). Eur J Cancer. 2014;50:1437-45.

18. Nishikawa K, Fujitani K, Inagaki H, Akamaru Y, Tokunaga S, Takagi M, et al. Randomised phase III trial of second-line irinotecan plus cisplatin versus irinotecan alone in patients with advanced gastric cancer refractory to $S-1$ monotherapy: TRICS trial. Eur J Cancer. 2015;51:808-16.

19. Stewart LA, Clarke M, Rovers M, Riley RD, Simmonds M, Stewart G, et al. Preferred reporting items for systematic review and meta-analyses of individual participant data: the PRISMAIPD statement. JAMA. 2015;313:1657-65.

20. Kawato Y, Aonuma M, Hirota Y, Kuga H, Sato K. Intracellular roles of $\mathrm{SN}-38$, a metabolite of the camptothecin 
derivative CPT-11, in the antitumor effect of CPT-11. Cancer Res. 1991;51:4187-91.

21. Iyer L, King CD, Whitington PF, Green MD, Roy SK, Tephly TR, et al. Genetic predisposition to the metabolism of irinotecan (CPT-11). Role of uridine diphosphate glucuronosyltransferase isoform 1A1 in the glucuronidation of its active metabolite (SN38) in human liver microsomes. J Clin Invest. 1998;101:847-54.

22. Gupta E, Lestingi TM, Mick R, Ramirez J, Vokes EE, Ratain MJ. Metabolic fate of irinotecan in humans: correlation of glucuronidation with diarrhea. Cancer Res. 1994;54:3723-5.

23. Innocenti F, Undevia SD, Iyer L, Chen PX, Das S, Kocherginsky $\mathrm{M}$, et al. Genetic variants in the UDP-glucuronosyltransferase 1A1 gene predict the risk of severe neutropenia of irinotecan. J Clin Oncol. 2004;22:1382-8.

24. Shitara K, Matsuo K, Mizota A, Kondo C, Nomura M, Takahari $\mathrm{D}$, et al. Association of fluoropyrimidines, platinum agents, taxanes, and irinotecan in any line of chemotherapy with survival in patients with advanced gastric cancer. Gastr Cancer. 2011;14:155-60.

25. Grothey A, Sargent D, Goldberg RM, Schmoll HJ. Survival of patients with advanced colorectal cancer improves with the availability of fluorouracil-leucovorin, irinotecan, and oxaliplatin in the course of treatment. J Clin Oncol. 2004;22:1209-14.

26. Yoshida K, Kodera Y, Kochi M, Ichikawa W, Kakeji Y, Sano T, et al. Addition of docetaxel to oral fluoropyrimidine improves efficacy in patients with stage III gastric cancer: interim analysis of JACCRO GC-07, a randomized controlled trial. J Clin Oncol. 2019;37:1296-304.

27. Bang YJ, Kim YW, Yang HK, Chung HC, Park YK, Lee KH, et al. Adjuvant capecitabine and oxaliplatin for gastric cancer after D2 gastrectomy (CLASSIC): a phase 3 open-label, randomised controlled trial. Lancet. 2012;379:315-21.

28. Fuse N, Bando H, Chin K, Ito S, Yoshikawa T, Tsuburaya A, et al. Adjuvant capecitabine plus oxaliplatin after D2 gastrectomy in Japanese patients with gastric cancer: a phase II study. Gastr Cancer. 2017;20:332-40.

29. Wilke H, Muro K, Van Cutsem E, Oh SC, Bodoky G, Shimada Y, et al. Ramucirumab plus paclitaxel versus placebo plus paclitaxel in patients with previously treated advanced gastric or gastrooesophageal junction adenocarcinoma (RAINBOW): a doubleblind, randomised phase 3 trial. Lancet Oncol. 2014;15:1224-355.

Publisher's Note Springer Nature remains neutral with regard to jurisdictional claims in published maps and institutional affiliations.

\section{Affiliations}

\section{Kazuhiro Nishikawa ${ }^{1}$ (D) Wasaburo Koizumi ${ }^{2} \cdot$ Akira Tsuburaya $^{3} \cdot$ Takeharu Yamanaka $^{4} \cdot$ Satoshi Morita $^{5}$. Kazumasa Fujitani $^{6} \cdot$ Yusuke Akamaru $^{7} \cdot$ Ken Shimada $^{8} \cdot$ Hisashi Hosaka $^{9}$. Norisuke Nakayama ${ }^{10}$. Toshimasa Tsujinaka $^{11}$ - Junichi Sakamoto ${ }^{12}$}

Wasaburo Koizumi

koizumi@med.kitasato-u.ac.jp

Akira Tsuburaya

tuburayaa@gmail.com

Takeharu Yamanaka

yamanaka@yokohama-cu.ac.jp

Satoshi Morita

smorita@kuhp.kyoto-u.ac.jp

Kazumasa Fujitani

fujitani@gh.opho.jp

Yusuke Akamaru

akamaru@ka3.so-net.ne.jp

Ken Shimada

shimakenken60@hotmail.com

Hisashi Hosaka

hosaka@gunma-cc.jp

Norisuke Nakayama

norisuke@ kcch.jp

Toshimasa Tsujinaka

tsujinaka@hosp.kaizuka.osaka.jp

Junichi Sakamoto

sakamjun@tokaihp.jp

1 Department of Surgery, National Hospital Organization Osaka National Hospital, 2-1-14, Houenzaka, Chuo-ku, Osaka 540-0006, Japan
2 Department of Gastroenterology, Kitasato University East Hospital, 2-1-1, Asamizodai, Minami-ku, Sagamihara, Kanagawa 252-0380, Japan

3 Department of Surgery, Ozawa Hospital, 1-1-17, Honcho, Odawara 250-0012, Japan

4 Department of Biostatistics, Yokohama City University School of Medicine, 3-9, Fukuura, Kanazawa-ku, Yokohama 236-0004, Japan

5 Department of Biomedical Statistics and Bioinformatics, Kyoto University Graduate School of Medicine, 54, Shogoinkawaharacho, Sakyo-ku, Kyoto 606-8397, Japan

6 Department of Surgery, Osaka General Medical Center, 3-1-56, Bandaihigashi, Sumiyoshi-ku, Osaka 558-0056, Japan

7 Department of Surgery, Ikeda Municipal Hospital, 3-1-18, Jyonan, Ikeda, Osaka 563-0025, Japan

8 Department of Internal Medicine, Division of Medical Oncology Showa University Koto Totosu Hospital, 5-1-38 Toyosu, Koto-ku, Tokyo 135-8577, Japan

9 Department of Gastroenterology, Gunma Prefectural Cancer Center, 617-1, Takahayashinishi-cho, Ohta 373-0828, Japan

10 Department of Gastroenterology, Kanagawa Cancer Center, 2-3-2, Nakao, Asahi-ku, Yokohama 241-0815, Japan

11 Department of Surgery, Kaizuka City Hospital, 3-10-20, Hori, Kaizuka 597-0015, Japan

12 Tokai Central Hospital, 4-6-2, Sohara Higashijimacho, Kakamigahara 504-8601, Japan 